PROCEEDINGS OF THE

AMERICAN MATHEMATICAL SOCIETY

Volume 126, Number 11, November 1998, Pages 3253-3256

S $0002-9939(98) 04612-7$

\title{
A SHORT PROOF FOR THE STABILITY THEOREM FOR POSITIVE SEMIGROUPS ON $L_{p}(\mu)$
}

\author{
LUTZ WEIS
}

(Communicated by Palle E. T. Jorgensen)

\begin{abstract}
We give a short proof showing that the growth bound of a positive semigroup on $L_{p}(\mu)$ equals the spectral bound of its generator. It is based on a new boundedness theorem for positive convolution operators on $L_{p}\left(L_{q}\right)$. We also give a counterexample, showing that Gearhart's result does not extend from Hilbert spaces to $L_{p}(\mu)$-spaces.
\end{abstract}

\section{The Results}

Let $T_{t}$ be a $c_{0}$-semigroup on $L_{p}(\Omega, \mu), 1 \leq p<\infty$, with generator $A$. $T_{t}$ is called positive if $f \geq 0$ implies $T_{t} f \geq 0$ for all $t$. The spectral bound $s(A)$ of $A$ is defined by

$$
s(A)=\sup \{\operatorname{Re} \lambda: \lambda \in \sigma(A)\}
$$

and the growth bound of $T_{t}$ is given by

$$
\omega\left(T_{t}\right)=\inf \left\{\omega: \exists C<\infty \text { with }\left\|T_{t}\right\| \leq C e^{\omega t} \text { for all } t \geq 0\right\} .
$$

The following theorem was proved in [9].

Theorem 1. If $T_{t}$ is a positive $c_{0}$-semigroup on $L_{p}(\Omega, \mu), 1 \leq p<\infty$, then $s(A)=$ $\omega\left(T_{t}\right)$.

The case $p=2$ is due to Gearhart and Greiner-Nagel, the case $p=1$ is due to Derndinger (see [7], [8], or [3], Theorems 9.5 and 9.7), but the general case remained an open problem for about 10 years. The proof in [9] used a new spectral mapping theorem for the evolutionary semigroup $I \otimes T_{t}$ on $L_{q}\left(L_{p}\right)$ by Latushkin and Montgomery-Smith [5] and an extrapolation procedure for the Yosida approximation of $T_{t}$. In [6] S. Montgomery-Smith simplified the proof by replacing the extrapolation procedure by a direct resolvent estimate.

In this note we give a new and simpler proof of Theorem 1 that is based on a boundedness result for positive convolutions on mixed norm spaces $L_{p}\left(L_{q}\right)$, and which may be of independent interest (see Theorem 2 below). With this convolution result we can reduce Theorem 1 to a well-known characterization of the spectral bound in terms of weak integrability ([3], Theorem 7.4).

Finally, we point out that recent counterexamples concerning stability of semigroups (see e.g. [1]) can be "transplanted" onto $L_{p}$-spaces. At the end of this note

Received by the editors July 22, 1996 and, in revised form, March 12, 1997.

1991 Mathematics Subject Classification. Primary 47D06.

Key words and phrases. Stability of semigroups, positive operators.

(C)1998 American Mathematical Society 
we give an example of a semigroup $T_{t}$ on $L_{p}(0,1), 1<p<\infty, p \neq 2$, for which $s(A)=s_{\infty}(A)<\omega\left(T_{t}\right)$. Here

$$
s_{\infty}(A)=\inf \{\omega: R(\lambda, A) \text { is uniformly bounded for } \lambda, \operatorname{Re} \lambda \geq \omega\} .
$$

This shows that Gearhart's spectral mapping theorem on Hilbert space (see e.g. [3], Theorem 9.6) does not extend to $L_{p}$-spaces for $1<p<\infty, p \neq 2$, and answers negatively question (IV) on page 147 of [8].

To state our convolution result we need the following notation. For $1 \leq p, q<\infty$ and $f \in L_{1, \text { loc }}\left(\mathbb{R}_{+} \times \Omega\right)$ put

$$
\begin{gathered}
\|f\|_{p, q}=\left(\int_{\Omega}\left(\int_{\mathbb{R}}|f(t, \omega)|^{q} d t\right)^{p / q} d \mu(\omega)\right)^{1 / p}, \\
L_{p}\left(L_{q}\right)=\left\{f \in L_{1, \text { loc }}\left(\mathbb{R}_{+}, \Omega\right):\|f\|_{p, q}<\infty\right\} .
\end{gathered}
$$

Note that for $p=q$ we have by Fubini's theorem $L_{p}\left(L_{p}\right)=L_{p}\left(\mathbb{R}, L_{p}(\Omega, \mu)\right)$. For these mixed norm spaces we have the following convolution result:

Theorem 2. For a fixed $1 \leq p<\infty$, let $t \in \mathbb{R} \rightarrow K(t)$ be a function of positive operators on $L_{p}(\Omega, \mu)$ such that $t \rightarrow K(t) f$ is locally Bochner integrable for $f \in$ $L_{p}(\Omega, \mu)$. Assume that for all $0 \leq h \in L_{p}(\Omega, \mu)$ and $0 \leq g \in L_{p^{\prime}}(\Omega, \mu)$ we have

$$
\int_{\mathbb{R}}\langle g, K(t) h\rangle_{L_{p}} d t \leq C\|g\|_{L_{p^{\prime}}} \cdot\|h\|_{L_{p}} .
$$

Then the convolution integral

$$
\mathcal{K} f(t)=\int_{-\infty}^{\infty} K(t-s)(f(s)) d s
$$

defined for stepfunctions $f: \mathbb{R} \rightarrow L_{p}(\Omega)$ extends to a bounded operator on $L_{p}\left(L_{q}\right)$ with $\|\mathcal{K} f\|_{p, q} \leq C\|f\|_{p, q}$ for all $1 \leq q \leq \infty$.

\section{The PROOFS}

Proof of Theorem 1. Since $s(A) \leq \omega\left(T_{t}\right)$ is always true, we only have to show that $s(A)<0$ implies $\omega\left(T_{t}\right)<0$, or, by a result of Pazy ([3], Proposition 9.4), that $s(A)<0$ implies that for all $f \in L_{p}(\Omega, \mu)$

$$
\int_{0}^{\infty}\left\|T_{t} f\right\|_{L_{p}}^{p} d t<\infty
$$

This claim can be reformulated as a convolution estimate. Indeed, for a fixed $\alpha>\omega\left(T_{t}\right)$

$$
\int_{0}^{t} T_{t-s}\left(e^{-\alpha s} T_{s} f\right) d s=\frac{1}{\alpha}\left(1-e^{-\alpha t}\right) T_{t} x .
$$

Put $K(t)=T_{t}$ for $t \geq 0$ and $K(t)=0$ for $t<\infty$, and $f(t)=e^{-\alpha t} T_{t} f$ for $t \geq 0$ and $f(t)=0$ for $t<0$. Then for $t \geq 1$ there is a constant $D$ such that

$$
\left\|T_{t} x\right\| \leq D\left\|\int_{-\infty}^{\infty} K(t-s)(f(s)) d s\right\| .
$$

The function $t \rightarrow K(t)$ satisfies the assumption of Theorem 2 since by Theorem 7.4 of [3] we have for all $0 \leq g \in L_{p^{\prime}}$ and $0 \leq f \in L_{p}$ that

$$
\int_{0}^{\infty}\left\langle g, T_{t} f\right\rangle d t \leq\|R(0, A)\|\|g\|_{L_{p^{\prime}}}\|f\|_{L_{p}} .
$$


Since $f \in L_{p}\left(\mathbb{R}, L_{p}(\Omega)\right)$ we obtain from Theorem 2

$$
\int\left\|\int K(t-s)(f(s)) d s\right\|_{L_{p}}^{p} d(t) \leq C \int\|f(s)\|_{L_{p}}^{p} d s \leq C_{1}\|f\|^{p} .
$$

Now (3) together with (2) implies (1) and the proof is complete. Alternatively, one can obtain (3) from the estimate in the proof of Theorem 1 in [6].

Proof of Theorem 2. First we check the claim for $q=1$. Given a stepfunction $f: \mathbb{R} \rightarrow L_{p}(\Omega)$ with $f \geq 0$ and a $0 \leq g \in L_{p^{\prime}}(\Omega)$ we have for all $N \in \mathbb{N}$

$$
\begin{aligned}
\left\langle g, \int_{-N}^{N} \mathcal{K} f(t) d t\right\rangle_{L_{p}} & =\int_{-N}^{N}\langle g, \mathcal{K} f(t)\rangle_{L_{p}} d t \\
& =\int_{-N}^{N}\left\langle g, \int K(s) f(t-s) d s\right\rangle d t \\
& =\iint_{-N}^{N}\langle g, K(s) f(t-s)\rangle d t d s \\
& =\int\left\langle g, K(s)\left[\int_{-N}^{N} f(t-s) d t\right]\right\rangle d s \\
& \leq \int\langle g, K(s) h\rangle d s \leq C\|\|_{L_{p^{\prime}}} \cdot\|h\|_{L_{p}}
\end{aligned}
$$

by assumption, where $h=\int f(t) d t$ with $\|h\|_{L_{p}}=\|f\|_{p, 1}$. Since such stepfunctions are dense in $L_{p}\left(L_{1}\right)$ we can extend $\mathcal{K}$ to $L_{p}\left(L_{1}\right)$ with

$$
\|\mathcal{K} f\|_{p, 1} \leq C\|f\|_{p, 1} \text {. }
$$

For $q=\infty$ and a stepfunction $f: \Omega \rightarrow L_{\infty}(\mathbb{R}), f(t, \omega)=\sum_{k} g_{k}(t) \chi_{A_{k}}(\omega)$, with $f \geq 0$ the integral

$$
\int_{-N}^{N} K(s) f(t-s) d s=\sum_{k} \int_{-N}^{N} g_{k}(t-s) K(s)\left[\chi_{A_{k}}(\omega)\right] d s
$$

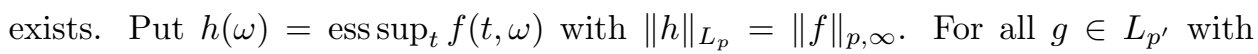
$g \geq 0,\|g\|_{L_{p^{\prime}}}=1$ and $N \in \mathbb{N}$ we have

$$
\begin{aligned}
\left\langle g, \int_{-N}^{N} K(s) f(t-s) d s\right\rangle & \leq\left\langle g, \int_{-N}^{N} K(s) h d s\right\rangle_{L_{p}} \leq \int\langle g, K(s) h\rangle d s \\
& \leq C\|g\|_{L_{p^{\prime}}} \cdot\|h\|_{L_{p}}=C\|f\|_{p, \infty} .
\end{aligned}
$$

Since stepfunctions with countably many values are dense in $L_{p}\left(L_{\infty}\right)$ we can extend $\mathcal{K}$ to $L_{p}\left(L_{\infty}\right)$ by Fatou's Lemma and continuity so that $\|\mathcal{K} f\|_{p, \infty} \leq C\|f\|_{p, \infty}$.

Interpolating in the scale $L_{p}\left(L_{q}\right), 1 \leq q \leq \infty$, gives the general claim according to [2], Theorem 5.1.2.

Example. Let $X=L_{p}(1, \infty) \cap L_{2}(1, \infty)$ with norm $\|f\|=\|f\|_{2}+\|f\|_{p}$ for $2<p<$ $\infty$. Consider the semigroup $\left(S_{t} f\right)(x)=f\left(x e^{t}\right), t \geq 0$, with generator $(B f)(x)=$ $x\left(\frac{d}{d x} f\right)(x)$ on a suitable domain and $(R(0, B) f)(x)=\int_{x}^{\infty} f(y) \frac{d y}{y}$. One can check that $s(B)=-\frac{1}{2}<-\frac{1}{p}=\omega\left(S_{t}\right)$ (cf. [1]). Since $S_{t}$ is positive, we also have $s_{\infty}(B)=s(B)($ see $[3]$, Corollary 7.5$)$. 
By [4], 2.e.8(ii) and section 2.f, there is an isomorphism $J$ of $X$ onto $L_{p}[0,1]$ (essentially given by a stochastic integral with respect to the Poisson process). Then the semigroup $T_{t}=J S_{t} J^{-1}$ on $L_{p}[0,1]$ with generator $A=J B J^{-1}$ on $D(A)=$ $J(D(B))$ still satisfies $s_{\infty}(A)=-\frac{1}{2}<-\frac{1}{p}=\omega\left(T_{t}\right)$.

If $1<p<2$ we take the dual of $T_{t}$ on $L_{p^{\prime}}$, to obtain a similar example.

\section{REFERENCES}

[1] W. Arendt, Spectrum and growth of positive semigroups, in Evolution Equations (G. Ferreyra, G. R. Goldstein and F. Neubrander, eds.), Lecture Notes in Pure and Applied Math., vol. 168, Marcel Dekker, 1994. MR 95m:47062

[2] J. Bergh and J. Löfström, Interpolation Spaces, Springer Verlag, 1976. MR 58:2349

[3] Ph. Clement, H. J. A. M. Heijmans, et al., One Parameter Semigroups, North-Holland, 1987. MR 89b: 47058

[4] J. Lindenstrauß and L. Tzafriri, Classical Banach Spaces, Vol. II, Springer Verlag, 1979. MR 81c:46001

[5] Y. Latushkin and S. J. Montgomery-Smith, Evolutionary semigroups and Lyapunov theorems in Banach spaces, Journal of Functional Analysis 127 (1995), 173-197. MR 96k:47072

[6] S. J. Montgomery-Smith, Stability and dichotomy of positive semigroups on $L_{p}$, Proc. Amer. Math. Soc. 124 (1996), 2433-2437. MR 96j:47037

[7] R. Nagel (ed.), One Parameter Semigroups of Positive Operators, Springer Verlag, 1986. MR 88i: 47022

[8] J. van Neerven, The Asymptotic Behavior of a Semigroup of Linear Operators, Birkhäuser Verlag, Basel, Boston, Berlin, 1996. MR 98d:47001

[9] L. Weis, The stability of positive semigroups on $L_{p}$ spaces, Proc. Amer. Math. Soc. 123 (1995), 3089-3094. MR 95m:47074

Universität Karlsruhe, Mathematisches Institut I, Postfach 69 80, Englerstrasse 2, 76128 KarlsRuhe, Germany

E-mail address: Lutz.Weis@math.uni-Karlsruhe.de 